\title{
A COMPARATIVE EVALUATION OF THE NUMBER OF STREPTOCOCCUS MUTANS COLONIES IN THE ENAMEL SURFACE OF TEETH AFTER THE APPLICATION OF CPP-ACP CONTAINING PROPOLIS AND CPP-ACP WITHOUT PROPOLIS
}

\author{
RISQA RINA DARWITA ${ }^{1 *}$, KEVIN SETIJONO² ${ }^{2}$, FADHILAH ALMASYHUR ${ }^{2}$, PETER ANDREAS ${ }^{1}$, ARIADNA A DJAIS ${ }^{3}$ \\ ${ }^{1}$ Department of Dental Public Health and Preventive Dentistry, Faculty of Dentistry, Universitas Indonesia. ${ }^{2}$ Dental Education Program, \\ Faculty of Dentistry, Universitas Indonesia. ${ }^{3}$ Department of Oral Biology, Faculty of Dentistry, Universitas Indonesia. \\ Email: risqarina2008@gmail.com
}

Received: 26 June 2020, Revised and Accepted: 06 July 2020

\section{ABSTRACT}

Objective: This study was to determine the efficacy of CPP-ACP combined with propolis by evaluating the number of Streptococcus mutans colonies in the enamel surfaces of children aged 7-9 years before and after the application of CPP-ACP containing propolis.

Methods: A quasi-experimental pretest-posttest design with purposive sampling was used in this study, which was conducted on 32 students aged 7-9 years who were divided into two groups that were intervention and control groups. Two young dentists examined the students, and their results were calibrated by a kappa value of 0.82 . The number of $S$. mutans colonies was evaluated at baseline, and after a CPP-ACP propolis paste was applied to white spot surfaces in the intervention group and CPP-ACP without propolis was applied to white spot surfaces of students in the control group in every day for 4 weeks.

Results: There was a significant decrease in the proportion of S. mutans colonies both of the intervention group (28\%) and the control group (26\%) $(\mathrm{p}<0.05)$. However, there was no significant difference in the effect of CPP-ACP propolis combination compared to CPP-ACP without propolis in decreasing the number of $S$. mutans colonies ( $p>0.05)$.

Conclusion: This study confirmed that CPP-ACP containing propolis is not better than CPP-ACP without propolis in decreasing the number of S. mutans colonies; however, the CPP-ACP propolis combination has the potential to be used as an alternative material to prevent demineralization and improve remineralization of white spots in enamel surfaces.

Keywords: Casein phosphopeptide-amorphous calcium phosphate, Remineralization, White spot, Propolis, Streptococcus mutans.

(c) 2020 The Authors. Published by Innovare Academic Sciences Pvt Ltd. This is an open access article under the CC BY license (http://creativecommons. org/licenses/by/4. 0/) DOI: http://dx.doi.org/10.22159/ijap.2020.v12s2.PP-25

\section{INTRODUCTION}

White spot lesion or early enamel lesion is a localized area in the enamel surfaces of teeth, which is characterized by its whiter shade with a more fragile structure than that of normal teeth. It is also considered as the initial process of dental caries [1]. This condition is caused due to an imbalance between the demineralization and remineralization processes of teeth, which causes the teeth to lose their ionic structure as well as the collapse of the enamel-forming hydroxyapatite crystals. At this stage, there is no cavity on the surface of the teeth, and the teeth can get restored to their original state by increasing the remineralization or the reformation of tooth enamel hydroxyapatite crystals.

One of the dental remineralization ingredients that are suitable to use and have been evaluated for efficacy is casein phosphopeptideamorphous calcium phosphate (CPP-ACP) [1]. CPP-ACP exhibits good remineralization ability and can, therefore, be used as an additional therapeutic material for the treatment of non-invasive early dental lesions as well as for maintaining good oral hygiene. CPP-ACP works by increasing the levels of calcium and phosphate ions in the oral cavity, which support the process of remineralization while stopping the process of demineralization that might be happening to the teeth.

Demineralization in the tooth enamel surface occurs due to several factors, and hence, it is also referred to as a multifactorial process. The formation of biofilms containing cariogenic bacteria such as Streptococcus mutans is the primary etiology of demineralization in the tooth enamel surface. Cariogenic bacteria can metabolize sucrose and produce acid, which trigger the collapse of the tooth hydroxyapatite crystals and lead to demineralization. Therefore, the use of dental demineralization agents also must be considered, one of it is an antibacterial agent, which might be used to reduce the number of $S$. mutans colonies to inhibit the process of demineralization [2]. One of the natural antibacterial ingredients that are commonly used is propolis, which has been widely used in several oral and dental health procedures such as disinfection in postsurgical wound canal treatment and intracanal irrigation and also as a pulp-capping material, mouthwash, and a cariostatic agent [3]. Propolis is a natural ingredient possessing antibacterial, antiviral, antiparasitic, and antifungal properties. It is also considered as a strong oxidative agent [4]. Therefore, it could have the potential to effectively reduce the number of cocci and Gram-positive bacteria (rod shaped). Propolis can inhibit the increase of bacterial colonization by destroying the cell wall of bacteria using flavonoids, the active ingredient of polyphenol compounds found in propolis [5].

The use of CPP-ACP as a remineralization agent containing propolis as an antibacterial agent indicates good potential, this is because of the nature of each ingredient that has been tested on dental health regarding the fact that its use in combination is not common. Therefore, to determine the remineralization effects of CPP-ACP paste containing propolis in increasing the process of tooth enamel remineralization, it is necessary to conduct a study analyzing the effect of CPP-ACP paste containing propolis in decreasing the number of $S$. mutans colonies in the tooth enamel surface. 


\section{MATERIALS AND METHODS}

Materials

The CPP-ACP paste containing propolis was used for intervention group, while the CPP-ACP paste withouth propolis is CPP-ACP tooth mouse from GC Corp., Asia which used for control group.

The sample is dental plaque which taken from the surface of whitespot enamel, then the total number of $S$. mutans was obtain from dental plaque. Trypticase Yeast Extract Cystine Sucrose Bacitracin (TYS20B) agar was used as the growth medium. Phosphate-buffered saline (PBS) is a storage media of $S$. mutans bacteria from dental plaque which put from white spot enamel surface before and after application of CPP-ACP paste containing propolis and CPP-ACP paste GC Tooth Mousse.

\section{Methods}

This study used a quasi-experimental pretest-posttest design with purposive sampling to determine the differences and the effects of applying CPP-ACP paste containing propolis and CPP-ACP without propolis in terms of the number of $S$. mutans colonies in the white spot enamel surfaces of 32 primary school students aged 7-9 years in Cipinang, East Jakarta. Ethical clearance was obtained from FKG UI Dentistry Research Ethics Committee (No. 44/Ethical Approval/ FKG UI/VI/2018). Reliability value was analyzed based on 2 times' measurement at two different time points and in five different study subjects. All the collected data were analyzed using the SPSS program, and a value of 0.82 indicated that the result belonged to good category in terms of reproducibility. This also implied that the performance of all operators was reliable and that they conducted the oral examination in a similar manner.

All the study respondents were divided into two groups, consist of intervention group of CPP-ACP propolis and control group of CPP-ACP without propolis (Tooth Mousse from GC Corp.). At baseline, the tooth surfaces of all the 32 students with white spots were checked and samples of $S$. mutans were obtained from dental plaque from the white spot enamel surface. Then, the CPP-ACP paste containing propolis was applied to the first group as an intervention group and CPP-ACP without propolis was applied to the control group in every day for 4 weeks (28 days). S. mutans colonies were obtained from dental plaques on the white spot enamel surface of all subjects.

\section{Bacterial culture}

The all bacterial samples of $S$. mutans were inoculated into TYS20B selective medium, then cultured and incubated in an aerobic atmosphere at $37^{\circ} \mathrm{C}$ for $48 \mathrm{~h}$, after that all S. mutans sample colonies were calculated after $2 \times 24 \mathrm{~h}$ using a colony counter in the Health Science Laboratory. Data were processed using the Microsoft Excel program, and both univariate and bivariate analyses were conducted using Wilcoxon and paired sample $t$-tests.

\section{Data collection process}

All students were examined using a standard dental instrument by a dentist, dental examination consists of dental plaque examination to get the dental plaque index was calculated using the Silness-Loe plaque index (1964) before and after the application of CPP-ACP containing propolis for the intervention group, wherein the control group students were treated with CPP-ACP Tooth Mousse from GC Corp. In the first dental examination conducted at baseline, the dental plaque examination and the application of CPP-ACP containing propolis and CPP-ACP Tooth Mousse were performed every morning around 8-9 am. This treatment is carried out for 28 days ( 4 weeks). The sampling of dental plaque in white spot enamel surface and saliva was done at the time of dental examination, then all dental plaques and saliva were placed into a $1 \mathrm{ml}$ tube, after which all subjects, the treatment group was treated with CPP-ACP containing propolis, and the control group was treated with CPP-ACP obtained from GC Corp. every day till 28 days. The number of $S$. mutans colonies was analyzed from all samples of dental plaques and saliva on the $1^{\text {st }}$ day of examination and on day of 28. The CPP-ACP was prepared according to the patent of the World Intellectual Property Organization proposed by Eric C. Reynolds with the US publication number 2005/0037948 calcium phosphopeptide complexes [6].

\section{RESULTS AND DISCUSSION}

Table 1 describes the total number of subjects in the intervention group and the control group. In the intervention group, only subjects aged 7 and 8 years participated, whereas in the control group, the subjects were aged 7 years. As shown in Table 1, only $56.25 \%$ of students were treated with CPP-ACP containing propolis, whereas in the control group, $87.5 \%$ of the students were treated with CPP-ACP without propolis.

Table 2 shows the results of comparison of the effect before and after the application of CPP-ACP containing propolis compared to that of CPPACP without propolis. There was a decrease in the number of $S$. mutans colonies after the application of both CPP-ACP containing propolis (418.94 CFU/ml) and CPP-ACP without propolis (376.25 CFU/ml), with the difference being $42.69 \mathrm{CFU} / \mathrm{ml}$. This difference in $S$. mutans colony number was not statistically significant $(\mathrm{p}=0.738)$ when analyzed by the unpaired $t$-test, which indicated that there was no significant

Table 1: Frequency distribution of the total number of subjects in the CPP-ACP paste containing propolis group ( $n=16)$ and the control group ( $n=16)$ treated with CPP-ACP paste without propolis according to age and sex

\begin{tabular}{|c|c|c|c|c|c|c|c|c|c|c|c|c|}
\hline \multirow[t]{3}{*}{ Group age (years) } & \multicolumn{6}{|c|}{ CPP-ACP containing propolis } & \multicolumn{6}{|c|}{ CPP-ACP without propolis } \\
\hline & \multicolumn{2}{|c|}{ Male } & \multicolumn{2}{|c|}{ Female } & \multicolumn{2}{|c|}{ Total } & \multicolumn{2}{|c|}{ Male } & \multicolumn{2}{|c|}{ Female } & \multicolumn{2}{|c|}{ Total } \\
\hline & $n$ & $\%$ & $n$ & $\%$ & $n$ & $\%$ & $n$ & $\%$ & $n$ & $\%$ & $n$ & $\%$ \\
\hline 7 & 2 & 12.5 & 5 & 31.25 & 7 & 43.75 & - & & - & & & \\
\hline 8 & 3 & 18.75 & 6 & 37.5 & 9 & 56.25 & 9 & 56.25 & 5 & 31.25 & 14 & 87.5 \\
\hline 9 & - & - & - & - & - & - & 1 & 6.25 & 1 & 6.25 & 2 & 12.5 \\
\hline Total & 5 & 31.25 & 11 & 68.75 & 16 & 100 & 10 & 62.5 & 6 & 37.5 & 16 & 100 \\
\hline
\end{tabular}

Table 2: Comparison between the effect of CPP-ACP paste containing propolis and CPP-ACP paste without propolis in decreasing the number of Streptococcus mutans colonies

\begin{tabular}{|c|c|c|c|}
\hline \multirow[t]{2}{*}{$\begin{array}{l}\text { Time } \\
\text { Group }\end{array}$} & \multirow{2}{*}{$\begin{array}{l}\text { Number of } S \text {. mutans before } \\
\text { application }\end{array}$} & \multirow{2}{*}{$\begin{array}{l}\begin{array}{l}\text { Number of } S . \text { mutans } \\
\text { after application }\end{array} \\
\text { Mean } \pm \text { SD }\end{array}$} & \multirow[t]{2}{*}{$\begin{array}{l}\text { Mean differences between } S \text {. mutans } \\
\text { colonies before and after application }\end{array}$} \\
\hline & & & \\
\hline $\begin{array}{l}\text { CPP-ACP propolis }(\mathrm{n}=16) \\
\text { CPP-ACP without propolis }(\mathrm{n}=16)\end{array}$ & $\begin{array}{l}1469.19 \pm 797.35 \\
1400.25 \pm 702.15\end{array}$ & $\begin{array}{l}1050.25 \pm 737.17 \\
1024.00 \pm 673.61\end{array}$ & $\begin{array}{l}418.94 \text { (decreasing) } \\
376.25 \text { (decreasing) }\end{array}$ \\
\hline
\end{tabular}


difference between the effect after the application of either CPP-ACP containing propolis CPP-ACP without propolis.

The significant differences in the number of $S$. mutans before and after application of CPP-ACP paste containing propolis could be correlated with the presence of propolis as an antibacterial agent with its ability that has been widely investigated in recent years. In an earlier study, Alves et al. (2007) investigated the effects of propolis extract in decreasing the number of $S$. mutans colonies in vivo [7]. They investigated 41 individuals by subjecting them to gargling water containing propolis extract and observed a significant decrease in the average number of S. mutans colonies in their study subjects. Another study conducted by Hegde et al. (2013) reported a statistically significant decrease in the number of $S$. mutans colonies in subjects who gargled water containing propolis [8].

A decrease in the number of $S$. mutans colonies might occur due to several propolis forming materials, one of which is flavonoid [9]. Flavonoids have been known to possess a broad range of biological capabilities, including antioxidant, antibacterial, antiviral, antifungal, and anti-inflammatory activities. Flavonoids present in propolis contain several vital ingredients, including apigenin and tt-farnesol, that contribute to their antibacterial properties [10]. Apigenin can inhibit plaque formation by inhibiting GTF enzyme activity, while tt-farnesol has good antibacterial properties and can inhibit the growth and metabolism of $S$. mutans by disrupting bacterial membrane formation.

In the present study, in the intervention group treated with CPP-ACP paste without propolis (Tooth Mousse from GC Corp.), S. mutans colony number decreased to an average value of $376.25 \mathrm{CFU} / \mathrm{ml}$. The number of $S$. mutans colonies before and after the application of CPP-ACP was assessed using the paired sample $t$-test, which resulted in $\mathrm{p}=0.002$, indicating a significant difference in the number of $S$. mutans colonies before and after the application of CPP-ACP paste without propolis.

The results of this study are consistent with those of some previous studies. For instance, Plonka et al. analyzed the effect of application of CPP-ACP paste in children aged 18-24 months every day and observed a significant decrease in the number of $S$. mutans colonies compared to that in the control group [11]. Similarly, another study conducted by Emamieh et al. evaluated the efficacy of chewing gum containing CPP-ACP as a major ingredient in comparison with xylitol-based gum in decreasing the number of $S$. mutans colonies [12]. They observed a decrease in S. mutans colony number in both groups; however, the use of CPP-ACP-based gum resulted in a significant decrease compared to that by the xylitol-based gum. These findings imply that CPP-ACP has an inhibitory effect on S. mutans present in the oral cavity and might affect its cariogenicity in vivo.

The decrease in the number of $S$. mutans colonies using CPP-ACP materials has been widely discussed in recent years. This decrease is related to the antibacterial ability of CPP-ACP, in addition to it being a reservoir of ions that are useful for teeth remineralization; it also binds to salivary pellicle receptors [13]. This binding with the salivary pellicle receptor then reduces the attachment of various bacteria, one of which is $S$. mutans. This concept has been tested in several studies, for example, the study by Schüpbach et al. who conducted an in vitro test using scanning electron microscope (SEM) to observe the nature of bacterial attachment after the application of CPP-ACP [14].

The efficacy of the CPP-ACP paste containing propolis and that of CPPACP without propolis in decreasing the number of $S$. mutans colonies could be roughly estimated based on the decrease in the mean $S$. mutans colony number between the two groups. In the CPP-ACP containing propolis group, there was a decrease of $418.94 \mathrm{CFU} / \mathrm{ml}$, and in the non-propolis group, there was a decrease of $376.25 \mathrm{CFU} / \mathrm{ml}$, indicating a higher decrease by $42.69 \mathrm{CFU} / \mathrm{ml}$ in the former group. However, when analyzed by the independent sample $t$-test, this decrease was no statistically significant $(\mathrm{p}=0.738)$.
Although the concept of using CPP-ACP containing propolis has not been widely investigated, there are few studies such as those by Sahlan and Lucia Purwanti (2016) and Soekanto et al. (2017) [15,16]. In the study of Sahlan, a combination of CPP-ACP and ethanolic extract propolis (EEP) was used to assess several variables, one among which was a decrease in the number of $S$. mutans colonies [16]. The authors observed decrease of $37.5 \%$ and $57.73 \%$ in the number of S. mutans colonies using the CPP-ACP paste combined with $2 \%$ EEP and 4\% EEP, respectively. However, in the study conducted by Soekanto et al., there was no significant difference in the decrease of S. mutans colony number with the application of the CPP-ACP paste containing or not containing propolis. All together, the present study has shown that CPP-ACP paste containing propolis has the potential to be used as an alternative for the use of CPP-ACP Tooth Mousse as a remineralization material that could inhibit the activity of biofilm formation, thereby decreasing the number of $S$. mutans colonies found on tooth surfaces. However, further studies are needed to obtain a clear understanding of the efficacy of CPP-ACP.

\section{CONCLUSION}

Application of both CPP-ACP paste containing propolis and CPP-ACP paste without propolis resulted in a decrease in the number of $S$. mutans colonies. No significant difference was observed in the decrease in the number of $S$. mutans colonies between the use of CPP-ACP paste containing propolis and without propolis. There was no significant difference in the decrease in the plaque index between the use of CPP-ACP paste containing propolis and without propolis. The overall results confirm that CPP-ACP paste containing propolis does not have better efficacy than CPP-ACP without propolis in reducing the number of $S$. mutans colonies and the plaque index; however, it can possibly be used as an alternative remineralization material.

\section{ACKNOWLEDGMENT}

The authors would like to acknowledge the Directorate of Higher Education and Research Technology grant.

\section{REFERENCES}

1. Al-Batayneh OB, Jbarat RA, Al-Khateeb SN. Effect of application sequence of fluoride and CPP-ACP on remineralization of white spot lesions in primary teeth: An in-vitro study. Arch Oral Biol 2017;83:236-40.

2. Decker EM, Klein C, Schwindt D, Von Ohle C. Metabolic activity of Streptococcus mutans biofilms and gene expression during exposure to xylitol and sucrose. Int J Oral Sci 2014;6:195-204.

3. Parolia A, Thomas M. Propolis and its potential uses in oral health. Int J Med Med Sci 2010;2:210-5.

4. Machorowska-Pieniazek A, Skucha-Nowak M, Mertas A, Tanasiewicz M, Niedzielska I, Morawiec T, et al. Effects of brazilian propolis on dental plaque and gingiva in patients with oral cleft malformation treated with multibracket and removable appliances: A comparative study. Evid Based Complement Alternat Med 2016;2016:2038407.

5. Vanni R, Waldner-Tomic NM, Belibasakis GN, Attin T, Schmidlin PR, Thurnheer T. Antibacterial efficacy of a propolis toothpaste and mouthrinse against a supragingival multispecies biofilm. Oral Health Prev Dent 2015;13:531-5.

6. Llena C, Ledya A, Forner L. CPP-ACP and CPP-ACFP versus fluoride varnish in remineralisation of early caries lesions. A prospective study. Eur J Pediatr Dent 2015;16:181-6.

7. Duailibe S, Gonçalves A, Ahid F. Effect of a propolis extract on Streptococcus mutans counts in vivo. J Appl Oral Sci 2007;15:420-3.

8. Hegde KS, Bhat SS, Rao A, Sain S. Effect of propolis on Streptococcus mutans counts: An in vivo study. Int J Clin Pediatr Dent 2013;6:22-5.

9. Hwu YJ, Lin FY. Effectiveness of propolis on oral health: A metaanalysis. J Nurs Res 2014;22:221-9.

10. Listyasari N, Santoso O. Inhibition of dental plaque formation by toothpaste containing propolis. Dent J 2012;45:208-11.

11. Plonka K, Pukallus M, Holcombe T, Barnett A, Walsh L, Seow W. Randomized controlled trial: A randomized controlled clinical trial comparing a remineralizing paste with an antibacterial gel to prevent early childhood caries. Pediatr Dent 2013;35:8-12.

12. Emamieh S, Khaterizadeh Y, Goudarzi H, Ghasemi A, Baghban A, Torabzadeh $\mathrm{H}$. The effect of two types chewing gum containing casein 
phosphopeptide-amorphous calcium phosphate and xylitol on salivary Streptococcus mutans. J Conserv Dent 2015;18:192.

13. Rose RK. Binding characteristics of Streptococcus mutans for calcium and casein phosphopeptide. Caries Res 2000;34:427-31.

14. Schüpbach P, Neeser JR, Golliard M, Rouvet M, Guggenheim B. Incorporation of caseinoglycomacropeptide and caseinophosphopeptide into the salivary pellicle inhibits adherence of mutans streptococci. J Dent Res 1996;75:1779-88.
15. Sahlan M, Purwanti L. Tooth spray with active ingredients casein phosphopeptide-amorphous calcium phosphate (CPP-ACP) and ethanolic extract propolis (EEP) to inhibit tooth demineralization. Commun Sci Technol 2016;1:56-60.

16. Soekanto SA, Duhita GC, Bachtiar EW, Sahlan M. The effect of CPPACP-propolis chewing gum on calcium and phosphate ion release on caries-active subjects' saliva and the formation of Streptococcus mutans biofilm. J Phys Conf Ser 2017;884:012137. 